TRANSACTIONS OF THE

AMERICAN MATHEMATICAL SOCIETY

Volume 357, Number 9, Pages 3789-3811

S 0002-9947(05)03721-9

Article electronically published on March 31, 2005

\title{
DIFFERENTIATION EVENS OUT ZERO SPACINGS
}

\author{
DAVID W. FARMER AND ROBERT C. RHOADES
}

\begin{abstract}
If $f$ is a polynomial with all of its roots on the real line, then the roots of the derivative $f^{\prime}$ are more evenly spaced than the roots of $f$. The same holds for a real entire function of order 1 with all its zeros on a line. In particular, we show that if $f$ is entire of order 1 and has sufficient regularity in its zero spacing, then under repeated differentiation the function approaches, after normalization, the cosine function. We also study polynomials with all their zeros on a circle, and we find a close analogy between the two situations. This sheds light on the spacing between zeros of the Riemann zeta-function and its connection to random matrix polynomials.
\end{abstract}

\section{INTRODUCTION}

If $f$ is a polynomial with all of its zeros on the real line, then all of the zeros of the derivative $f^{\prime}$ also lie on the real line. The same holds for entire functions of order 1 which are real on the real line. But while the zeros of $f$ in an interval could in principle be any finite subset of the interval, the condition that $f$ have only real zeros will impose restrictions on the zeros of $f^{\prime}$. The main idea of this paper is that if the zeros of $f$ lie on a line, then the zeros of $f^{\prime}$ are more evenly spaced than the zeros of $f$. Repeated differentiation leads the zeros to become more and more evenly spaced, and under appropriate conditions the zeros will approach equal spacing. See Section 2.4 for precise statements.

There is a long history to the behavior of the zeros of entire functions under repeated differentiation. Polya [16] made several interesting conjectures for the case of real entire functions, some of which have only been solved recently [5, 6. 10 11, 12, 17]. Many of those questions concern the reality of zeros of a real entire function, or the motion of zeros toward (or away from) the real line when the function is differentiated. In this paper we take a complementary view and consider the motion along the real line when the function is differentiated.

1.1. Zeros of the derivative. It is tempting to think of the zeros of $f^{\prime}$ as lying close to the midpoint of neighboring zeros of $f$. That is a useful model, and we analyze the sequence of midpoints and use it as a tool to study the zeros of the derivative. A more accurate picture is to think of the zeros of $f^{\prime}$ as trying to move as far as possible from the zeros of $f$, and so move toward regions where there are fewer zeros of $f$. See Figure 1 for an example. If $z_{j}<z_{j+1}$ are consecutive zeros of $f$, then Rolle's theorem asserts that there will be a zero $z^{\prime}$ of $f^{\prime}$ between $z_{j}$ and

Received by the editors October 21, 2003 and, in revised form, March 25, 2004.

2000 Mathematics Subject Classification. Primary 30C15.

Research of the first author was supported by the American Institute of Mathematics and the NSF.

(C)2005 American Mathematical Society 
$z_{j+1}$. If there are a large number of other zeros near $z_{j+1}$, then $z^{\prime}$ will be closer to $z_{j}$.

There are several intuitive reasons for this phenomenon. Suppose $z_{j}$ are the zeros of $f$, listed in increasing order, and consider

$$
\frac{f^{\prime}}{f}(z)=\sum_{k} \frac{1}{z-z_{k}}
$$

If $z_{j}<p<z_{j+1}$, then $f^{\prime}(p)=0$ if and only if $\frac{f^{\prime}}{f}(p)=0$. Substituting $z=p$ into (1.1.1) we see two kinds of terms: positive for $k \leq j$, and negative for $k \geq j+1$. If there are not many zeros near $z_{j+1}$, then all of those negative terms have to be balanced by something. If there are not many zeros near $z_{j}$, then the only option is moving $p$ closer to $z_{j}$. One can think of $M=\frac{f^{\prime}}{f}\left(\frac{1}{2}\left(z_{j}+z_{j+1}\right)\right)$ as a measure of how far the zero of $f^{\prime}$ will be from the midpoint. Specifically,

$$
p \approx \begin{cases}\frac{1}{2}\left(z_{j}+z_{j+1}\right)+\frac{M}{8}\left(z_{j+1}-z_{j}\right)^{2} & \text { if } M \text { is small, } \\ \left(z_{j} \text { or } z_{j+1}\right)-\frac{1}{M} & \text { if } M \text { is large. }\end{cases}
$$

An illustrative example is the polynomial $f$ with zeros at $\{0,1, \ldots, N\}$. The first zero of the derivative is very close to $1 / \log N$, and $\left(f^{\prime} / f\right)\left(\frac{1}{2}\right) \approx-\log N$.

A less explicit version of the above explanation uses Gauss' electrostatic model. By (1.1.1), the zeros of $f^{\prime}$ can be thought of as points of zero electric field for a collection of equal charges located at $\left\{z_{k}\right\}$, where like charges repel according to an inverse linear law. If there is a region of high concentration of charges, then these will push the equilibrium points toward regions of lower charge concentration.

Another approach concerns the relationship between the spacing of zeros and the size of the relative maxima and minima of $f$. Roughly speaking, $f$ gets big when there are larger than average sized gaps between zeros. But since $f$ is a polynomial with all zeros on a line, it cannot change slope too fast. This forces the locations of the relative maxima to shift around so that the slope of $f$ can change appropriately. See Figure1, particularly near the first local minimum and the third local maximum.

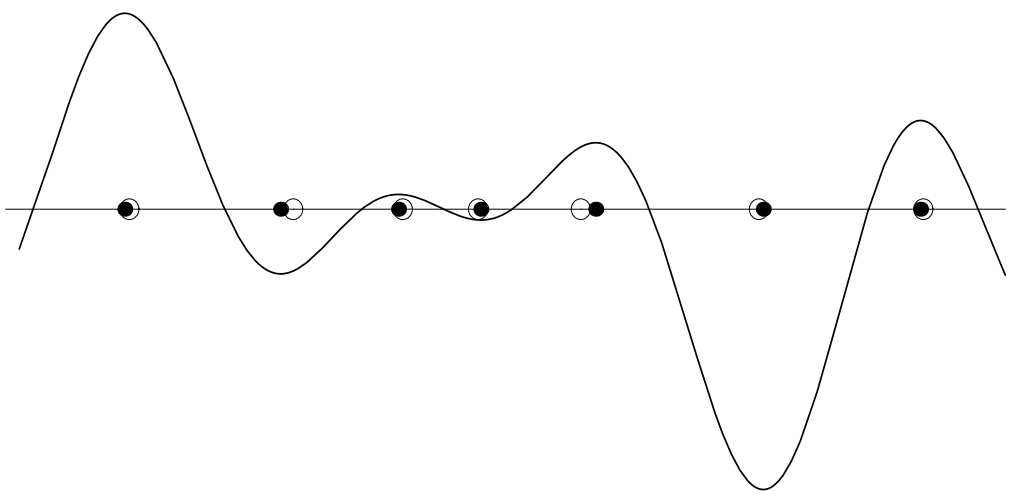

Figure 1. The circles are the midpoints of neighboring zeros of the polynomial $f$, and the dots are the zeros of $f^{\prime}$. Think of the circles as a target, which the zeros of $f^{\prime}$ often miss. 
This approach is interesting because the current view is that the spacing of zeros is the key to understanding the large values of functions like the Riemann zeta-function.

In addition to entire functions with zeros on a line, we also consider polynomials with all their zeros on a circle. We find a close analogy between the two cases. The motivation here is that much recent work has concerned the connection between $L$ functions (such as the Riemann $\zeta$-function, which conjecturally have all their zeros on a line) and the characteristic polynomials of random unitary matrices (which have all their zeros on the unit circle). The connection between random matrices and $L$-functions is discussed extensively in [3].

In the next section we start with an old result that motivates the remainder of the paper. We first consider polynomials, and then generalize to entire functions of order 1. Our main results are stated in Section 2.4. In Section 3 we consider a somewhat simpler averaging process which we later will compare to differentiation. In Section 4 we consider the case of zeros which are almost equally spaced, and determine the rate at which differentiation evens out the spacing. Note that in Sections 3 and 4 we treat in parallel the cases of zeros on a line and zeros on a circle. In Section 5 we complete the proof of our main results, and also discuss the case that zeros do not lie on a line.

We thank Robin Chapman, Brian Conrey, Nathan Feldman, Chris Hughes, and Paul McGuire for helpful conversations, and the referee for several useful suggestions.

\section{Small gaps Become larger}

If $f$ is a function with all its zeros on a line, define the "smallest gap between zeros" function

$$
G[f]=\inf _{j \neq k}\left|a_{j}-a_{k}\right|,
$$

where $a_{j}$ and $a_{k}$ are distinct zeros of $f$, with zeros repeated according to their multiplicity (so $G[f]=0$ if $f$ has a multiple zero). We will show that if $f$ is a polynomial with all zeros on a line, then $G\left[f^{\prime}\right] \geq G[f]$. That is, the smallest gap between zeros of $f^{\prime}$ is at least as large as the smallest gap between zeros of $f$. This is in keeping with the underlying principle that zeros of $f^{\prime}$ tend to "move away" from regions with higher density of zeros of $f$, and to "move toward" regions with lower density of zeros of $f$.

2.1. Motivating result. The following theorem is attributed to M. Riesz; see [18]. The result was rediscovered several times [19, 20, 21], including some results which are quantitatively stronger than the statement below. Our proof is similar in spirit, but somewhat simpler, than previous versions.

Theorem 2.1.1. Let $f(x)=e^{\alpha x} h(x)$, where $h$ is a polynomial with only real zeros. If $\alpha$ and $a$ are real, then $G\left[f^{\prime}+a f\right] \geq G[f]$, with strict inequality provided the zeros of $f$ are simple.

In particular, zeros of $f^{\prime}$ are 'further apart' than zeros of $f$.

It is implicit, and we give Lagrange's proof following equation (2.1.2) below, that all zeros of $f^{\prime}+a f$ are real. 
Proof. We may assume that $f$ has only simple zeros $a_{0}<a_{1}<\cdots<a_{J}$. We can write

$$
f(x)=e^{\alpha x} \prod_{j=0}^{J}\left(x-a_{j}\right)
$$

and so

$$
\frac{f^{\prime}+a f}{f}(x)=\alpha+a+\sum_{j=0}^{J} \frac{1}{x-a_{j}} .
$$

We see that all the zeros of $f^{\prime}+a f$ are real because if $x$ has positive (negative) imaginary part, then each term in the sum has negative (positive) imaginary part. Also, between each pair of zeros of $f$ there is exactly one zero of $f^{\prime}+a f$, and there is one additional zero if $a+\alpha \neq 0$.

Let $q<p$ be successive zeros of $f^{\prime}+a f$. Assume, for a contradiction, that $p-q \leq a_{j+1}-a_{j}$ for all $j$. Note that this is equivalent to

$$
\frac{1}{p-a_{j+1}} \geq \frac{1}{q-a_{j}}
$$

because $p-a_{j+1}$ and $q-a_{j}$ are either both positive or both negative, which follows from the fact that there is only one zero of $f$ between $p$ and $q$.

We have

$$
0=\alpha+a+\sum_{j=0}^{J} \frac{1}{p-a_{j}}
$$

and

$$
0=\alpha+a+\sum_{j=0}^{J} \frac{1}{q-a_{j}} .
$$

Subtracting the two equations gives

$$
\begin{aligned}
0 & =\sum_{j=0}^{J}\left(\frac{1}{p-a_{j}}-\frac{1}{q-a_{j}}\right) \\
& =\frac{1}{p-a_{0}}-\frac{1}{q-a_{J}}+\sum_{j=0}^{J-1}\left(\frac{1}{p-a_{j+1}}-\frac{1}{q-a_{j}}\right) .
\end{aligned}
$$

The first two terms above are strictly positive, and by (2.1.3) all the terms in the sum are nonnegative. So the right side is strictly positive, which is a contradiction.

Repeatedly applying the result leads to various linear combinations of $f$ and its derivatives which satisfy the corresponding inequality. For example, $G\left[f-c f^{\prime \prime}\right] \geq$ $G[f]$ provided $c \geq 0$. 
2.2. Entire functions of order 1. We wish to generalize the above result to functions having infinitely many zeros. The appropriate class to consider is that of entire functions of order at most 1 . We recall the standard terminology and properties of these functions. For more details, see [1].

If $f$ is an entire function, then $f$ is of order $\rho$ if

$$
\limsup _{r \rightarrow \infty} \frac{\log \log M(r)}{\log r}=\rho,
$$

where $M(r)=\max _{|z|=r}|f(z)|$. That is, $f(z)=O\left(\exp \left(|z|^{\rho+\delta}\right)\right)$ for all $\delta>0$ and no $\delta<0$.

If $f$ is of order $\rho$, then $f$ has type $\tau$ if

$$
\limsup _{r \rightarrow \infty} r^{-\rho} \log M(r)=\tau .
$$

That is, $f(z)=O\left(\exp \left((\tau+\delta)|z|^{\rho}\right)\right)$ for all $\delta>0$ and no $\delta<0$. We say that $f$ has minimal (respectively, finite or maximal) type if $\tau=0$ (respectively, $0<\tau<\infty$ or $\tau=\infty)$.

For example, $\cos (z)=\frac{1}{2}\left(e^{i z}+e^{-i z}\right)$, so $\cos (z)$ has order 1 and type 1 . And by Stirling's formula, $1 / \Gamma(z)$ has order 1 and maximal type.

Of particular interest is the connection between order, type, and the distribution of zeros. Let $\left(z_{n}\right)$ denote the zeros of $f$, with $z_{j} \leq z_{j+1}$ and zeros repeated according to their multiplicity. Denote by $n(r)$ the number of zeros of $f$ in $|z| \leq r$. If $f$ has only real zeros, then let $n_{+}(r)$ (respectively, $n_{-}(r)$ ) denote the number of zeros in $(0, r]$ (respectively, $[-r, 0))$. A characterization of functions of finite type is due to Lindelöf:

Proposition 2.2.1 (Lindelöf). If $\rho$ is an positive integer, then the entire function $f$ of order $\rho$ is of finite type if and only if $n(r)=O\left(r^{\rho}\right)$ and the sum over zeros

$$
S(r)=\sum_{\left|z_{n}\right| \leq r} z_{n}^{-\rho}
$$

is bounded.

That $\cos (z)$ has finite type and $1 / \Gamma(z)$ has maximal type follows directly from Lindelöf's theorem.

The Riemann $\zeta$-function is not entire because it has a simple pole at $s=1$, but $(s-1) \zeta(s)$ is entire. From the Dirichlet series representation $\zeta(s)=\sum_{n=1}^{\infty} n^{-s}$ it follows that the $\zeta$-function is bounded for $\Re(s)>1+\delta$. By the functional equation

$$
\zeta(s)=2^{s} \pi^{s-1} \sin \left(\frac{1}{2} s \pi\right) \Gamma(1-s) \zeta(1-s)
$$

and the fact that the sine and $\Gamma$-function have order 1 , it follows that the $\zeta$-function has order 1.

The Riemann $\zeta$-function has maximal type because $\zeta(s)$ has zeros at the negative even integers and $\frac{1}{2 \pi} T \log (T / 2 \pi e)+O(\log T)$ zeros with imaginary part $0<t<T$ and real part $0<\sigma<1$, so it fails both tests in Lindelöf's theorem.

The Riemann $\xi$-function is given by

$$
\xi(s)=\frac{1}{2} s(s-1) \pi^{-\frac{1}{2} s} \Gamma\left(\frac{1}{2} s\right) \zeta(s) .
$$

It satisfies the functional equation $\xi(s)=\xi(1-s)$. The $\xi$-function is entire of order 1 and has the same nonreal zeros as the $\zeta$-function, so it has maximal type. 
The Riemann Hypothesis is the assertion that all zeros of the $\xi$-function have real part $\frac{1}{2}$.

Sometimes it is convenient to use the Riemann $\Xi$-function, given by $\Xi(z)=$ $\xi\left(\frac{1}{2}+i z\right)$. The $\Xi$-function is an even function which is real on the real axis, is entire of order 1 and maximal type. Assuming the Riemann Hypothesis, $\Xi$ has only real zeros and $n_{+}(r)=n_{-}(r)=(r / 2 \pi) \log (r / 2 \pi e)+O(\log r)$.

In the generalization of Theorem 2.1.1 to entire functions of order 1 we will make use of the Hadamard product. Suppose $f$ is entire of order $\rho$ and let $N=[\rho]$ be the largest integer less then or equal to $\rho$. Then there exists a polynomial $Q$ of degree at most $N$ such that

$$
f(z)=e^{Q(z)} z^{n_{0}} \prod_{n}\left(1-\frac{z}{z_{n}}\right) \exp \left(P_{N}\left(\frac{z}{z_{n}}\right)\right),
$$

where $P_{N}(z)=z+\frac{1}{2} z^{2}+\cdots+\frac{1}{N} z^{N}$ and $n_{0}$ is the order of the zero of $f(z)$ at $z=0$.

2.3. Generalization of Theorem 2.1 .1 to entire functions of order 1 . We have

Theorem 2.3.1. Suppose $f$ is an entire function of order 1 which is real on the real axis and has only real zeros. If $q<p$ are consecutive zeros of $f^{\prime}+a f, a \in \mathbb{R}$, then

$$
\inf z_{n+1}-z_{n} \leq p-q \leq \sup z_{n+1}-z_{n} .
$$

If the zeros of $f$ are simple and equality holds for one of the inequalities, then it also holds for the other, and $f(z)=A e^{B z} \cos (C z+D)$ for some real $A, B, C$, and $D$.

So, if the zeros of $f$ are not equally spaced, then differentiation makes the smallest gaps larger and the largest gaps smaller. It might not be obvious that if the zeros of $f$ are equally spaced, then the same holds for $f^{\prime}$, but one can check that the set of functions of the form $A e^{B z} \cos (C z+D)$ is closed under differentiation. In particular, the factor $e^{B x}$ shifts all of the zeros of the derivative of $\cos (C x+D)$ by the same amount.

Proof. We may assume that $f(0) \neq 0$ and the zeros of $f$ are simple. We have the Hadamard factorization

$$
f(z)=A e^{B z} \prod_{n}\left(1-\frac{z}{z_{n}}\right) \exp \left(\frac{z}{z_{n}}\right)
$$

so

$$
\frac{f^{\prime}+a f}{f}(z)=a+B+\sum_{n} \frac{1}{z-z_{n}}-\frac{1}{z_{n}} .
$$

Imitating the previous proof, the terms $a, B$, and $z_{n}$ cancel and equation (2.1.4) becomes

$$
0=\sum_{j} \frac{1}{p-z_{j+1}}-\frac{1}{q-z_{j}} .
$$

By (2.1.3) every term in the sum is nonnegative, and we have a contradiction unless $p-q=z_{j+1}-z_{j}$ for all $j$. This proves the left-hand inequality in the theorem. The proof of the other inequality is identical.

Finally, if $p-q=z_{j+1}-z_{j}$ for all $j$, then the zeros of $f$ are equally spaced, so we recognize the Hadamard product of $f$ to be of the stated form. 
A shortcoming of Theorem 2.3 .1 is that the inequalities can be vacuous. For example, the Riemann $\Xi$-function has $\inf z_{n+1}-z_{n}=0$. To formulate a "local" version of Theorem 2.3.1 we must be able to speak of the "local density" of zeros. Suppose

$$
n_{ \pm}(r)=L(r)+E(r)
$$

where $L(r)$ is a nice function and $E(r)=o(L(r))$ should be thought of as an error term. Then $L^{\prime}(r)$ is the density of zeros near $r$, meaning that $L^{\prime}(r)^{-1}$ is the average gap between zeros near $r$. We want to show that if $f^{\prime}$ has zeros which are closer together than the average spacing, then in some neighborhood $f$ will also have zeros which are closer together than average. The following theorem asserts that the neighborhood can be taken to be of size $E(r) / L^{\prime}(r)$.

Theorem 2.3.2. Suppose $f$ is an entire function of order 1 which is real on the real axis, has only real zeros, and $n_{ \pm}(r)$ satisfies (2.3.5), where $L$ is an increasing smooth function such that $L^{(j)}(r) \ll r^{-j} L(r)$. Suppose $\kappa<K<1$ and $q<p$ are consecutive zeros of $f^{\prime}+a f, a \in \mathbb{R}$, such that $p-q<\kappa L^{\prime}(q)^{-1}$. Then there exist zeros $z_{n}, z_{n+1}$ of $f$ such that $\left|z_{n}-q\right| \ll E(q) / L^{\prime}(q)$ and $z_{n+1}-z_{n}<K L^{\prime}(q)^{-1}$.

For the Riemann $\Xi$-function we have $L(r)=(2 \pi)^{-1} r \log (r / 2 \pi e)$ and $E(r)=$ $\log r$, giving the following corollary.

Corollary 2.3.3. Suppose $0<\kappa<K<1$ and $\gamma_{1}^{\prime}<\gamma_{2}^{\prime}$ are consecutive zeros of $\Xi^{(n)}$ with $\gamma_{2}^{\prime}-\gamma_{1}^{\prime}<2 \pi \kappa / \log \gamma_{1}$. Then there exist consecutive zeros $\gamma_{1}<\gamma_{2}$ of $\Xi$ with $\left|\gamma_{1}-\gamma_{1}^{\prime}\right| \ll 1$ such that $\gamma_{2}-\gamma_{1}<2 \pi K / \log \gamma$.

The corollary is motivated by the problem of Landau-Siegel zeros. Conrey and Iwaniec [4] have shown that if there exists $\kappa<\frac{1}{2}$ and $\delta>0$ such that $\gamma_{2}-\gamma_{1}<$ $2 \pi \kappa / \log \gamma$ for $\gg T \log ^{\delta} T$ pairs of zeros $0<\gamma_{2}, \gamma_{1}<T$ of the $\Xi$-function, then one can find an effective lower bound for the class number of the imaginary quadratic field $\mathbb{Q}(\sqrt{-d})$. By the corollary, if we had $\gg T \log ^{\delta} T$ pairs of zeros $\gamma_{2}^{\prime}-\gamma_{1}^{\prime}<$ $2 \pi K / \log \gamma$ for $\Xi^{(n)}$, for any fixed $n>0$, then we would have $\gg T$ such pairs of zeros for $\Xi$. This falls short of the requirements for Conrey and Iwaniec's result. However, it may be possible to modify the proof of Theorem 2.3 .2 to show that either $\Xi$ has a pair of zeros which are closely separated and very near to $q$, or else $\Xi$ has a large number of zeros which are not too far from $q$.

Proof of Theorem 2.3.2. For a contradiction, suppose $z_{j+1}-z_{j}>p-q$ for $\left|q-z_{j}\right|<$ $X$. We can reuse everything in the previous proof up to (2.3.4), say

$$
\begin{aligned}
0 & =\sum_{j}\left(\frac{1}{p-z_{j+1}}-\frac{1}{q-z_{j}}\right) \\
& =\left(\sum_{\left|q-z_{j}\right| \leq X}+\sum_{q-z_{j}<-X}+\sum_{z_{j}-q>X}\right)\left(\frac{1}{p-z_{j+1}}-\frac{1}{q-z_{j}}\right) \\
& =S_{1}+S_{2}+S_{3} .
\end{aligned}
$$


By assumption, $S_{1} \geq 0$. We now estimate $S_{3}$, the treatment of $S_{2}$ being identical. Note that

$$
\begin{aligned}
\frac{1}{p-z_{j+1}}-\frac{1}{q-z_{j}} & =\frac{1}{q-z_{j+1}}-\frac{1}{q-z_{j}}+\frac{p-q}{\left(p-z_{j+1}\right)\left(q-z_{j+1}\right)} \\
& =\frac{1}{q-z_{j+1}}-\frac{1}{q-z_{j}}+\frac{p-q}{\left(q-z_{j+1}\right)^{2}}+O\left(\frac{(p-q)^{2}}{\left(q-z_{j+1}\right)^{2}}\right) .
\end{aligned}
$$

Let $Z_{J}$ denote the smallest zero of $f$ in $[q+X, \infty)$, so the sum in $S_{3}$ begins with $z_{J}$. In the sum $S_{3}$, the first two terms in (2.3.7) telescope, giving

$$
S_{2}=\frac{1}{z_{J}-q}+(p-q)\left(1+O\left(L^{\prime}(q)^{-1}\right) \sum_{z_{j}-q>X} \frac{1}{\left(q-z_{j+1}\right)^{2}} .\right.
$$

Writing the sum as a Steiltjes integral and integrating by parts we have

$$
\begin{aligned}
& \sum_{z_{j}-q>X} \frac{1}{\left(q-z_{j+1}\right)^{2}}=\int_{z_{J+1}}^{\infty} \frac{d n_{+}(r)}{(r-q)^{2}} \\
& \quad=\int_{z_{J+1}}^{\infty} \frac{L^{\prime}(r)}{(r-q)^{2}} d r+\int_{z_{J+1}}^{\infty} \frac{d E(r)}{(r-q)^{2}} \\
& \quad=\frac{L^{\prime}\left(z_{J+1}\right)}{z_{J+1}-q}+\frac{E(q)}{\left(z_{J+1}-q\right)^{2}}+\int_{z_{J+1}}^{\infty} \frac{L^{\prime \prime}(r)}{r-q} d r+2 \int_{z_{J+1}}^{\infty} \frac{E(r)}{(r-q)^{3}} d r \\
& \quad=(1+o(1)) \frac{L^{\prime}\left(z_{J+1}\right)}{z_{J+1}-q}+\frac{E\left(z_{J+1}\right)}{\left(z_{J+1}-q\right)^{2}} .
\end{aligned}
$$

In the last step we used $E(r)=o(L(r))$ and $L^{\prime \prime}(r) \ll L^{\prime}(r) / r$.

Adding all the terms and using the fact that $L^{\prime}\left(z_{J+1}\right) \sim L^{\prime}(q)$ and $E\left(z_{J+1}\right) \sim$ $E(q)$ we have

$$
S_{3} \sim \frac{1}{z_{J}-q}-\frac{(q-p) L^{\prime}(q)}{z_{J+1}-q}-\frac{E(q)(q-p)\left(z_{J+1}-q\right)^{-1}}{z_{J+1}-q} .
$$

We assumed $q-p \leq \kappa L^{\prime}(q)^{-1}$, so the first two terms sum to something larger than $\delta /\left(z_{J+1}-q\right)$ for some $\delta>0$. The numerator of the last term is bounded by $\kappa E(q) L^{\prime}(q)^{-1} X^{-1}$ which is smaller than $\delta$ provided $X>\delta^{-1} \kappa E(q) L^{\prime}(q)^{-1}$. For such $X$ we have $S_{3}>0$, which gives a contradiction. We have $X \ll E(q) / L^{\prime}(q)$, as claimed.

2.4. Long-term behavior. As described in the previous section, differentiation causes the small gaps between zeros of $f$ to become larger and the large gaps to become smaller. Thus, one might expect that under repeated differentiation the zeros of $f^{(n)}$ will approach equal spacing, which would equal the average spacing between zeros of $f$. Also, if the zeros of $f^{(n)}$ approach equal spacing, then $f^{(n)}(z)$ should approach $A e^{B z} \cos (C z+D)$, where $A, B, C$, and $D$ may depend on $n$.

In order for the above argument to hold, there must be an appropriate sense of average spacing between zeros of $f$. Below we show that it is sufficient to have $n_{+}(r) \sim n_{-}(r) \sim \kappa r$ for some $\kappa>0$, but it is possible that this condition can be weakened. Since $A e^{B z} \cos (C z+D)$ has finite type, one might expect that this would also be a necessary condition on $f$, but this is not the case. The assumptions in the following result are stronger than the first condition in Lindelöf's theorem, but weaker than the second. 
Theorem 2.4.1. Suppose $f$ is an entire function of order 1 which is real on the real axis, has only real zeros, and $n_{+}(r) \sim n_{-}(r) \sim \kappa r$. Then there exist sequences $\left(A_{n}\right),\left(B_{n}\right)$, and $\left(D_{n}\right)$ with $D_{n}$ bounded, such that

$$
\lim _{n \rightarrow \infty} A_{n} e^{B_{n} z} f^{(n)}\left(\kappa^{-1} z+D_{n}\right)=\cos (\pi z),
$$

uniformly for $|z| \leq X$ for any fixed $X>0$. In particular, the zeros of $f^{(n)}$ approach equal spacing.

We give the proof in Section 5 ,

It seems that $A_{n}$ in the theorem is increasing or decreasing according to whether $n(r)-2 \kappa r$ is generally negative or positive, but we have not succeeded in finding a precise statement. The theorem also suggests that the regularity in the distribution of the zeros of $f$ should lead to a great regularity in the Taylor series coefficients of $f$. This seems worth exploring further.

A trivial example of the theorem is when $f(z)=A e^{B z} \cos (z+D)$ for some $A, B, D$. We have $f^{\prime}(z)=\sqrt{B^{2}+1} f(z+\varphi)$, where $\cos (\varphi)=B / \sqrt{B^{2}+1}$ and $\sin (\varphi)=1 / \sqrt{B^{2}+1}$, Therefore, the conclusion of the theorem holds with $A_{n}=$ $A^{-1}\left(B^{2}+1\right)^{-\frac{n}{2}}$ and $D_{n} \equiv-D-n \varphi(\bmod 2 \pi)$. Somewhat more generally, suppose $f(z)=A e^{B z}(\cos (z+D)+E)$, where $|E| \leq 1$. This function has zeros consisting of two interlaced sequences which separately are equally spaced. The conclusion of the theorem holds with the same $A_{n}$ and $D_{n}$ as in the previous example. Similarly, if $f(z)=F(z) \cos (z)$ where $F$ is a degree $N$ polynomial, then the conclusion holds with $A_{n}=n^{-N}$ and $D_{n} \in\left\{-\frac{\pi}{2}, 0, \frac{\pi}{2}, \pi\right\}$.

A less trivial example is the Bessel function $J_{n}(z)$. We have $n_{ \pm}(r)=\pi^{-1} r+O(1)$, so the theorem applies. In this case the theorem can be verified directly because

$$
J_{n}(\pi z)=\frac{1}{\pi} \int_{0}^{\pi} \cos (\pi z \sin t-n t) d t
$$

so for an even number of derivatives

$$
\begin{aligned}
J_{n}^{(2 k)}(\pi z) & =\frac{(-\pi)^{k}}{\pi} \int_{0}^{\pi} \sin ^{2 k}(t) \cos (\pi z \sin t-n t) d t \\
& \sim(-\pi)^{k} \sqrt{\frac{\pi}{k}} \cos \left(\pi z-\frac{n \pi}{2}\right),
\end{aligned}
$$

because $\sqrt{k / 2 \pi} \sin ^{k} t d t$ is approaching a Dirac $\delta$-function at $t=\pi / 2$. So the conclusion of the theorem holds with $A_{2 k}=\pi^{-k} \sqrt{k / \pi}$ and $D_{2 k} \equiv \frac{n \pi}{2} \bmod 2 \pi$, and similarly for $k$ odd. Equation (2.4.2) can also be obtained from the Taylor series for $J_{n}$.

Theorem 2.4.1 asserts that the zeros of $f^{(n)}$ approach equal spacing. The approach to equal spacing is actually quite fast. The general case is somewhat intractable, so we will assume that the zeros are close to equally spaced and do a first order approximation. We also suppose further that $f$ is approximately an odd function, although this is not essential and we describe the necessary modifications during the proof. Thus,

$$
f(z)=C\left(z-\varepsilon_{0}\right) \prod_{j \neq 0}\left(1-\frac{z}{j+\varepsilon_{j}}\right) \exp \left(\frac{z}{j+\varepsilon_{j}}\right),
$$

where $\left|\varepsilon_{j}\right|<\varepsilon$ for some small $\varepsilon$. 
Theorem 2.4.2. Suppose $f$ is given in (2.4.3) and let $z_{n}^{j}$ be the zeros of the $j$ th derivative $f^{(j)}$. Then $z_{n+1}^{j}-z_{n}^{j}=1+O\left(\varepsilon j^{-1}\right)$.

We give the proof in Section 4.3

If the zeros of $f$ do not have a nonzero average spacing, then the results in this section do not apply. Suppose, for example, $n_{+}(r)$ and $n_{-}(r)$ grow faster than linearly. The "overall" average spacing is 0 , and under repeated differentiation there is a competition between zeros moving toward the origin and zeros trying to become locally equally spaced. If $n_{+}(r)$ and $n_{-}(r)$ are sufficiently nice, then there is the possibility that an analogue of Theorem 2.4.1 may hold. This was established by Haseo Ki [9] in the special case of the Riemann $\Xi$-function:

Theorem 2.4.3 (Haseo Ki 9]). There exist sequences $A_{n}$ and $C_{n}$, with $C_{n} \rightarrow 0$ slowly, such that

$$
\lim _{n \rightarrow \infty} A_{n} \Xi^{(2 n)}\left(C_{n} z\right)=\cos (z)
$$

uniformly on compact subsets of $\mathbb{C}$.

The above result was conjectured in an earlier verision of this paper. The $\Xi$ function has $n_{ \pm}(r)=(2 \pi)^{-1} r \log (r / 2 \pi e)+O(\log r)$ so the zero counting function is particularly well behaved. However, Ki's proof makes use of the fact that the $\Xi$ function has a nice representation as a Fourier transform, rather than directly using properties of the zeros. The theorem suggests that $\log \left(\xi^{(n)}\left(\frac{1}{2}\right)\right)$ should grow very regularly and not too much faster than linearly as $n \rightarrow \infty$. Rick Kreminski [13, 14 ] has calculated the first 490 derivatives and his data appears to grow approximately like $n \log n$.

More generally we conjecture:

Conjecture 2.4.4. Suppose $f$ is a real entire function of order 1 having only real zeros, with $n_{+}(r)$ and $n_{-}(r)$ sufficiently nice and $n_{+}(r)-n_{-}(r)$ not too large. Then there exist sequences $A_{n}, B_{n}, C_{n}$, and $D_{n}$ with $D_{n} / C_{n}$ bounded, such that

$$
\lim _{n \rightarrow \infty} A_{n} e^{B_{n} z} f^{(2 n)}\left(C_{n} z+D_{n}\right)=\cos (z)
$$

uniformly on compact subsets of $\mathbb{C}$.

\section{Averaging, instead of Differentiating}

Differentiation is a process which takes one sequence of points (the zeros of $f$ ) and replaces it with another sequence of points (zeros of $f^{\prime}$ ), such that the two sequences interlace each other. For comparison, we also consider the much simpler process of making a new sequence from the midpoints of neighboring elements of the given sequence.

3.1. Averaging on the line. Suppose $\left(x_{n}\right)$ is an increasing sequence. Then form new sequences $\left(x_{n}^{j}\right)$ where $x_{n}^{0}=x_{n}$ and $x_{n}^{j+1}=\frac{1}{2}\left(x_{n}^{j}+x_{n+(-1)^{j}}^{j}\right)$. That is, the terms of each new sequence are the midpoints of consecutive terms in the previous sequence. The subscript $n+(-1)^{j}$ is designed so that if $\left(x_{n}^{j}\right)$ is equally spaced, then $x_{n}^{j+2}=x_{n}^{j}$. If that subscript was $n+1$, then the equal spaced case would give $x_{n}^{j+2}=x_{n-1}^{j}$, and the terms would be "drifting to the right." 
Theorem 3.1.1. Suppose $\left(x_{n}\right)_{n \in \mathbb{Z}}$ is a sequence with $x_{n}=n+\varepsilon_{n}$, where $\varepsilon_{n} \ll$ $E(|n|)$, as $n \rightarrow \pm \infty$, for some increasing function $E$. If $E(n)=o(n)$, that is $x_{n} \sim n$, then as $j \rightarrow \infty$ the $j$ th midpoint sequence $\left(x_{n}^{j}\right)$ approaches equal spacing. Furthermore, if $E(n) \ll n^{\theta}$ with $0 \leq \theta<1$, then

$$
x_{n+1}^{j}-x_{n}^{j}=1+O\left(\left(n^{\theta}+j^{\frac{1}{2} \theta}\right) j^{-\frac{1}{2}}\right) .
$$

Proof. It is straightforward to show by induction that

$$
x_{n}^{j}=2^{-j} \sum_{0 \leq k \leq j}\left(\begin{array}{l}
j \\
k
\end{array}\right) x_{n+[j / 2]-k} .
$$

Thus,

$$
\begin{aligned}
x_{n+1}^{j}-x_{n}^{j}= & 2^{-j} \sum_{0 \leq k \leq j}\left(\begin{array}{l}
j \\
k
\end{array}\right)\left(x_{n+1+[j / 2]-k}-x_{n+[j / 2]-k}\right) \\
= & 2^{-j} \sum_{0 \leq k \leq j}\left(\begin{array}{l}
j \\
k
\end{array}\right)+2^{-j} \sum_{0 \leq k \leq j}\left(\begin{array}{l}
j \\
k
\end{array}\right)\left(\varepsilon_{n+1+[j / 2]-k}-\varepsilon_{n+[j / 2]-k}\right) \\
= & 1+2^{-j} \sum_{0 \leq k \leq j} \varepsilon_{n+1+[j / 2]-k}\left(\left(\begin{array}{l}
j \\
k
\end{array}\right)-\left(\begin{array}{c}
j \\
k-1
\end{array}\right)\right) \\
& +2^{-j}\left(\varepsilon_{n+[j / 2]-j}-\varepsilon_{n+[j / 2]}\right) \\
= & 1+2^{-j} \sum_{-\frac{j}{2} \leq \ell \leq \frac{j}{2}} \varepsilon_{n+\ell}\left(\begin{array}{c}
j \\
\frac{j}{2}+\ell
\end{array}\right) \frac{-\ell+1}{\frac{j}{2}-\ell+1}+O\left(2^{-j}(n+j)\right) .
\end{aligned}
$$

We may assume that $E$ is an increasing function and $E(a+b) \ll E(a)+E(b)$ for $a, b>0$. So the sum in (3.1.1) is bounded by

$$
\sum_{0 \leq \ell \leq \frac{j}{2}}(E(n)+E(\ell))\left(\begin{array}{c}
j \\
\frac{j}{2}+\ell
\end{array}\right) \frac{\ell}{\frac{j}{2}-\ell+1} .
$$

Now use the fact that the binomial distribution approaches the Gaussian to estimate the binomial coefficient

$$
\left(\begin{array}{l}
j \\
k
\end{array}\right) \sim \frac{2^{j+\frac{1}{2}}}{\sqrt{\pi j}} \exp \left(-\frac{2\left(k-\frac{j}{2}\right)^{2}}{j}\right) .
$$

So,

$$
\begin{aligned}
\sum_{0 \leq \ell \leq \frac{j}{2}} E(\ell)\left(\begin{array}{c}
j \\
\frac{j}{2}+\ell
\end{array}\right) \frac{\ell}{\frac{j}{2}-\ell+1} & \ll \frac{2^{j}}{j^{\frac{1}{2}}} \sum_{0 \leq \ell \leq \frac{j}{2}} E(\ell) \exp \left(-\frac{2 \ell^{2}}{j}\right) \frac{\ell}{\frac{j}{2}-\ell+1} \\
& \ll \frac{2^{j}}{j^{\frac{1}{2}}} \int_{0}^{\frac{j}{2}} E(t) \exp \left(-\frac{2 t^{2}}{j}\right) \frac{t}{\frac{j}{2}-t+1} d t \\
& =2^{j} j^{\frac{1}{2}} \int_{0}^{\frac{\sqrt{j}}{2}} E(t \sqrt{j}) \exp \left(-2 t^{2}\right) \frac{t}{\frac{j}{2}-t \sqrt{j}+1} d t .
\end{aligned}
$$


We may assume $E(a b) \ll E(a) E(b)$ for $a, b>1$, so (3.1.4) is bounded by

$$
2^{j} j^{\frac{1}{2}} E(\sqrt{j}) \int_{0}^{\frac{\sqrt{j}}{2}} \exp \left(-2 t^{2}\right) \frac{t^{2}}{\frac{j}{2}-t \sqrt{j}+1} d t \ll 2^{j} j^{-\frac{1}{2}} E(\sqrt{j}),
$$

the integral being seen to be $\ll j^{-1}$ by breaking it at $j^{\delta}$, for any $0<\delta<\frac{1}{4}$, and estimating the two parts separately.

To finish the proof, note that if $E(n)=o(n)$, then $j^{-\frac{1}{2}} E(\sqrt{j})=o(1)$, and if $E(n) \ll n^{\theta}$, then the other estimates follows immediately.

If $x_{n}=n+O(1)$, then the above shows that the $j$ th midpoint sequence approaches equal spacing with a discrepancy of order $j^{-\frac{1}{2}}$. This convergence to equal spacing is actually quite slow, and in some sense it is the slowest way to "even out" a sequence. In particular, Theorem 2.4.2 says taking successive derivatives evens out the sequence much faster, with a discrepancy of order $j^{-1}$. These topics are discussed in Section 4.3 .

3.2. Averaging on the circle. As mentioned in the Introduction, it is believed that there is a close analogy between $L$-functions, which (conjecturally) have all their zeros on a line, and the characteristic polynomials of random unitary matrices, which have all their zeros on a circle. See [3]. It is obvious that the analogy must break down if pushed too far, for a polynomial has only finitely many zeros. In this section we consider the averaging of points on a circle, in analogy to the averaging on a line in the previous section. Here we find that the discrepancy from equal spacing vanishes exponentially, in sharp contrast to the $j^{-\frac{1}{2}}$ of the linear case. This exponential convergence is a general property of iterative averaging procedures on finite sets, as described in Section 4.4 of [7].

Suppose $p_{1}, \ldots, p_{\ell}$ are points on the unit circle, and form new sets of points on the circle $\left(p_{n}^{j}\right)$ in analogy to the midpoint sequences of the previous section, where $0 \leq p_{n}^{j}<2 \pi$ is interpreted as an angle. If $j<n$, then this process is indistinguishable from the averaging process in the previous section. But when $j>n$ the fact that there are only $n$ points on a circle comes into play. This causes the averaging to even out the spacing more rapidly.

Proposition 3.2.1. Let $\left(p_{n}\right)$ and $\left(p_{n}^{j}\right)$ be sequences of $\ell$ points on the unit circle as described above. Then $p_{n+1}^{j}-p_{n}^{j}=\frac{2 \pi}{\ell}+O\left(\left(1-\frac{\pi^{2}}{2 \ell^{2}}\right)^{j}\right)$.

Proof. Starting exactly as before, but using the fact that $p_{j}=p_{k}$ if $j \equiv k \bmod \ell$, we have

$$
\begin{aligned}
p_{n+1}^{j}-p_{n}^{j} & =2^{-j} \sum_{0 \leq k \leq j}\left(\begin{array}{l}
j \\
k
\end{array}\right)\left(p_{n+1+[j / 2]-k}-p_{n+[j / 2]-k}\right) \\
& =2^{-j} \sum_{m=1}^{\ell}\left(p_{m+1}-p_{m}\right) \sum_{\substack{0 \leq k \leq j \\
k \equiv M \bmod \ell}}\left(\begin{array}{l}
j \\
k
\end{array}\right),
\end{aligned}
$$

where $M=n+1+[j / 2]-m$.

Now let $\rho$ be a primitive $\ell$ th root of 1 , and let $\rho_{j}=\rho^{j}$ for $0 \leq j \leq \ell-1$. Using the binomial theorem and the fact that $\sum_{k} \rho_{k}^{n}=0$ except when $n \equiv 0 \bmod \ell$, we 
have

$$
\begin{aligned}
\ell \sum_{\substack{0 \leq k \leq j \\
k \equiv M \bmod \ell}}\left(\begin{array}{l}
j \\
k
\end{array}\right) & =(1+1)^{j}+\rho_{M_{1}}\left(1+\rho_{1}\right)^{j}+\cdots+\rho_{M_{\ell-1}}\left(1+\rho_{\ell-1}\right)^{j} \\
& =2^{j}+O\left(2^{j} \ell\left(1-\frac{\pi^{2}}{2 \ell^{2}}\right)^{j}\right)
\end{aligned}
$$

where $\rho_{M_{j}}$ is an ordering of the $\rho_{k}$ depending on $M$. The last step follows from $\left|1+e^{2 \pi i / \ell}\right|=2\left(1-\frac{\pi^{2}}{2 \ell^{2}}\right)+O\left(\ell^{-4}\right)$.

Finally, use the fact that $\sum_{m=1}^{\ell}\left(p_{m+1}-p_{m}\right)=2 \pi$ to finish the proof.

\section{Almost equally spaced zeros}

Suppose $f(z)$ is an entire function of order 1 which is real on the real axis, has only real zeros, and the zeros have average spacing 1 . As we repeatedly differentiate, the zeros will approach equal spacing, and we want to determine the rate at which this occurs. We will see that the approach to equal spacing is much faster than in the midpoint process of Section 3 .

We treat in detail the case that the zeros of $f$ are close to equally spaced, both on the line and on the circle. At the end of this section we determine the rate at which differentiation evens out zero spacing.

\subsection{Almost equally spaced zeros on a line. Suppose}

$$
f(z)=C\left(z-\varepsilon_{0}\right) \prod_{j \neq 0}\left(1-\frac{z}{j+\varepsilon_{j}}\right) \exp \left(\frac{z}{j+\varepsilon_{j}}\right),
$$

where $\left|\varepsilon_{j}\right|<\varepsilon$ for some small $\varepsilon$. That is, $f$ is approximately an odd function with zeros close to equally spaced.

Theorem 4.1.1. In the notation above, $f^{\prime}$ has zeros at $k+\frac{1}{2}+\alpha_{k}$, where

$$
\alpha_{k}=\frac{4}{\pi^{2}} \sum_{j} \frac{\varepsilon_{j+k}}{(2 j-1)^{2}}+O\left(\varepsilon^{2}\right),
$$

and $f^{\prime \prime}$ has zeros at $k+\beta_{k}$, where

$$
\beta_{k}=\frac{1}{3} \varepsilon_{k}+\frac{2}{\pi^{2}} \sum_{j \neq 0} \frac{\varepsilon_{j+k}}{j^{2}}+O\left(\varepsilon^{2}\right) .
$$

In the proof of Theorem 4.1.1 we will require the following standard formulas:

$$
\sum_{j} \frac{1}{(2 j+1)^{2}}=\frac{\pi^{2}}{4} \quad \text { and } \quad \sum_{j} \frac{1}{(2 j+1)^{2}(2(j+n)+1)^{2}}= \begin{cases}\frac{\pi^{2}}{8 n^{2}}, & n \neq 0 \\ \frac{\pi^{4}}{48}, & n=0 .\end{cases}
$$

Proof of Theorem 4.1.1. We have

$$
\frac{f^{\prime}}{f}(z)=\sum_{j}^{\prime} \frac{1}{z-j-\varepsilon_{j}}+\frac{1}{j+\varepsilon_{j}}
$$

Here and below, $\sum^{\prime}$ means that the $j=0$ term must be modified in the obvious way. The zeros of $f^{\prime}(z)$ will be approximately halfway between the zeros of $f$, so suppose one of the zeros is $k+\frac{1}{2}+\alpha_{k}$. 
Note: if we did not assume that $f$ was odd, and so a factor of $e^{a z}$ occurs in our function, then the zeros of $f^{\prime}$ would not be halfway between the zeros of $f$. But the zeros of $f^{\prime}$ would all be shifted from the midpoint by the same amount, and all of the calculations below would work with a slight modification. So,

$$
\begin{aligned}
0 & =\frac{f^{\prime}}{f}\left(k+\frac{1}{2}+\alpha_{k}\right) \\
& =\sum_{j}^{\prime} \frac{1}{k+\frac{1}{2}+\alpha_{k}-j-\varepsilon_{j}}+\frac{1}{j+\varepsilon_{j}} \\
& =\sum_{j}^{\prime} \frac{2}{1+2(k-j)}+\frac{4\left(\varepsilon_{j}-\alpha_{k}\right)}{(1+2(k-j))^{2}}+\frac{1}{j+\varepsilon_{j}}+O\left(\varepsilon^{2}+\alpha_{k}^{2}\right) \\
& =\sum_{j} \frac{4\left(\varepsilon_{j}-\alpha_{k}\right)}{(1+2(k-j))^{2}}+O\left(\varepsilon^{2}+\alpha_{k}^{2}\right) \\
& =4 \sum_{j} \frac{\varepsilon_{j+k}}{(2 j-1)^{2}}-\pi^{2} \alpha_{k}+O\left(\varepsilon^{2}+\alpha_{k}^{2}\right) .
\end{aligned}
$$

Solving for $\alpha_{k}$ gives the first formula in the theorem. The $O\left(\varepsilon^{2}\right)$ error term follows because the above formula shows that $\alpha_{k}=O\left(\varepsilon+\varepsilon^{2}+\alpha_{k}^{2}\right)$, but $\varepsilon^{2}=o(\varepsilon)$ and $\alpha_{k}^{2}=o\left(\alpha_{k}\right)$, so $\alpha_{k}=O(\varepsilon)$.

To understand the effect of the second derivative on the zeros, we must iterate the above formula. The second derivative $f^{\prime \prime}$ will have zeros near the integers, so suppose there is a zero at $k+\beta_{k}$. By the first formula in the theorem, and using formulas 4.1.2,

$$
\begin{aligned}
\beta_{k} & =\frac{4}{\pi^{2}} \sum_{j} \frac{\alpha_{j+k-1}}{(2 j-1)^{2}}+O\left(\varepsilon^{2}\right) \\
& =\frac{4}{\pi^{2}} \sum_{j} \frac{1}{(2 j-1)^{2}} \frac{4}{\pi^{2}} \sum_{m} \frac{\varepsilon_{m+j+k-1}}{(2 m-1)^{2}}+O\left(\varepsilon^{2}\right) \\
& =\frac{16}{\pi^{4}} \sum_{n} \varepsilon_{n+k} \sum_{j} \frac{1}{(2 j-1)^{2}} \frac{1}{(2(n-j+1)-1)^{2}}+O\left(\varepsilon^{2}\right), \\
& =\frac{1}{3} \varepsilon_{k}+\frac{2}{\pi^{2}} \sum_{n \neq 0} \frac{\varepsilon_{n+k}}{n^{2}}+O\left(\varepsilon^{2}\right)
\end{aligned}
$$

as claimed.

4.2. Almost equally spaced zeros on a circle. If the polynomial $f$ has all its zeros on a circle, then $f^{\prime}$ has all its zeros strictly inside the circle, except at points where $f$ has a multiple zero. This follows from the famous Gauss-Lucas theorem that the zeros of $f^{\prime}$ lie inside the convex hull of the zeros of $f$. Thus, if we are to find an analogy with the case of zeros on a line, we must do something slightly different than differentiation.

Lemma 4.2.1. If $f$ is a degree $n$ polynomial with all its zeros on the unit circle, then

$$
f^{j}(z):=\left(z \frac{d}{d z}\right)^{j} z^{-\frac{n}{2}} f(z)
$$

also has all its zeros on the unit circle. The zeros of $f^{j+1}$ interlace the zeros of $f^{j}$. 
Proof. From

$$
f(z)=\sum_{0 \leq k \leq n} a_{k} z^{k}=a_{n} \prod_{0 \leq k \leq n}\left(z-z_{j}\right)
$$

and the fact that $1 / z_{j}=\overline{z_{j}}$, we have $f(z)=\left(a_{0} / a_{n}\right) z^{n} \overline{f(1 / \bar{z})}$. It follows that $Z(z):=\left(a_{0} / a_{n}\right)^{-\frac{n}{2}} z^{-\frac{n}{2}} f(z)$ is real on the unit circle. By Rolle's theorem, $\frac{d}{d \theta} Z\left(e^{i \theta}\right)$ has $n$ zeros in $0 \leq \theta<2 \pi$. But these give the $n$ zeros of $Z^{\prime}$, which are the same as the zeros of $f^{1}$.

We now derive the analogue of Theorem 4.1.1 Suppose the zeros of $f$ are close to equally spaced on the unit circle:

$$
f(z)=\prod_{j}\left(z-e\left(\frac{j}{n}+\varepsilon_{j}\right)\right),
$$

where $\left|\varepsilon_{j}\right| \leq \varepsilon$ and we set $e(x)=e^{2 \pi i x}$. In the above formula and in Proposition 4.2.2, unrestricted sums and products over $j$ should be interpreted as over $j$ modulo $n$, and we consider $\varepsilon_{j}$ to only depend on $j$ modulo $n$.

Let

$$
g(z)=z^{-\frac{n}{2}} f(z)
$$

and suppose $g^{\prime}$ has a zero at $e\left(\frac{k}{n}+\frac{1}{2 n}+\alpha_{k}\right)$.

Proposition 4.2.2. In the notation above,

$$
\alpha_{k}=\frac{1}{n^{2}} \sum_{j} \frac{\varepsilon_{j+k}}{\sin ^{2}\left(\frac{\pi}{2} \frac{2 j-1}{n}\right)}+O\left(\varepsilon^{2}\right) .
$$

Note that the above sum is

$$
\sim \frac{4}{\pi^{2}} \sum_{j=-\infty}^{\infty} \frac{\varepsilon_{j+k}}{(2 j-1)^{2}}
$$

as $n \rightarrow \infty$, so this formula matches that in Theorem 4.1.1.

Corollary 4.2.3. $\zeta(2)=\frac{\pi^{2}}{6}$.

Proof. Using $\sin (x)^{-2}=x^{-2}+O(1)$, from Proposition 4.2 .2 we have

$$
\begin{aligned}
\alpha_{k} & =\frac{4}{\pi^{2}} \sum_{-\frac{n}{2}<j \leq \frac{n}{2}} \frac{\varepsilon_{j+k}}{(2 j-1)^{2}}+O\left(\frac{1}{n^{2}} \sum_{-\frac{n}{2}<j \leq \frac{n}{2}}\left|\varepsilon_{j+k}\right|\right)+O\left(\varepsilon^{2}\right) \\
& =\frac{4}{\pi^{2}} \sum_{-\frac{n}{2}<j \leq \frac{n}{2}} \frac{\varepsilon_{j+k}}{(2 j-1)^{2}}+O\left(\frac{\varepsilon}{n}\right)+O\left(\varepsilon^{2}\right),
\end{aligned}
$$

as $n \rightarrow \infty$ and $\varepsilon \rightarrow 0$.

If $\varepsilon_{j}=\varepsilon$ for all $j$, then by a change of variables we see that the zeros of $g^{\prime}$ are at $e\left(\frac{k}{n}+\frac{1}{2 n}+\varepsilon\right)$. This means that $\alpha_{k}=\varepsilon$, so canceling $\varepsilon$ in (4.2.2) gives

$$
1=\frac{4}{\pi^{2}} \sum_{-\frac{n}{2}<j \leq \frac{n}{2}} \frac{1}{(2 j-1)^{2}}+O\left(\frac{1}{n}\right)+O(\varepsilon) .
$$

Since $n$ and $\varepsilon$ are arbitrary, $1=\frac{4}{\pi^{2}} \sum_{j} \frac{1}{(2 j-1)^{2}}$, which is equivalent to $\sum_{j=1}^{\infty} \frac{1}{j^{2}}=$ $\frac{\pi^{2}}{6}$. 
Proof of Proposition 4.2.2 We have

$$
\frac{g^{\prime}}{g}(z)=-\frac{n}{2 z}+\sum_{j} \frac{1}{z-e\left(\frac{j}{n}+\varepsilon_{j}\right)},
$$

so setting $z=e\left(\frac{k}{n}+\frac{1}{2 n}+\alpha_{k}\right)$ gives

$$
\frac{n}{2 e\left(\frac{k}{n}+\frac{1}{2 n}\right) e\left(\alpha_{k}\right)}=\sum_{j} \frac{1}{e\left(\frac{k}{n}+\frac{1}{2 n}\right) e\left(\alpha_{k}\right)-e\left(\frac{j}{n}\right) e\left(\varepsilon_{j}\right)} .
$$

Write $E(t)=e(t)-1$, so that it is easier to keep track of the main terms:

$$
\begin{aligned}
\frac{n}{2} e\left(-\frac{k}{n}-\frac{1}{2 n}\right)+\frac{n}{2} e\left(-\frac{k}{n}-\frac{1}{2 n}\right) E\left(-\alpha_{k}\right) \\
=\sum_{j} \frac{1}{e\left(\frac{k}{n}+\frac{1}{2 n}\right)-e\left(\frac{j}{n}\right)+e\left(\frac{k}{n}+\frac{1}{2 n}\right) E\left(\alpha_{k}\right)-e\left(\frac{j}{n}\right) E\left(\varepsilon_{j}\right)} \\
=\sum_{j} \frac{1}{e\left(\frac{k}{n}+\frac{1}{2 n}\right)-e\left(\frac{j}{n}\right)}+\sum_{j} \frac{e\left(\frac{j}{n}\right) E\left(\varepsilon_{j}\right)-e\left(\frac{k}{n}+\frac{1}{2 n}\right) E\left(\alpha_{k}\right)}{\left(e\left(\frac{k}{n}+\frac{1}{2 n}\right)-e\left(\frac{j}{n}\right)\right)^{2}} \\
\quad+O\left(\sum_{j} \frac{\left|E\left(\varepsilon_{j}\right)\right|^{2}+\left|E\left(\alpha_{k}\right)\right|^{2}}{\left|e\left(\frac{k}{n}+\frac{1}{2 n}\right)-e\left(\frac{j}{n}\right)\right|^{3}}\right) .
\end{aligned}
$$

The first terms on both sides of the above can be canceled, because both equal

$$
\frac{d}{d z} \log \left(z^{n}-1\right)=\frac{n z^{n-1}}{z^{n}-1}=\sum_{j=1}^{n} \frac{1}{z-e(j / n)}
$$

evaluated at $e\left(\frac{k}{n}+\frac{1}{2 n}\right)$.

Rearranging to put the terms with $\alpha_{k}$ on one side and multiplying by $e\left(\frac{k}{n}+\frac{1}{2 n}\right)$, we have

$\frac{n}{2} E\left(-\alpha_{k}\right)+E\left(\alpha_{k}\right) \sum_{j} \frac{e\left(\frac{k}{n}+\frac{1}{2 n}\right)^{2}}{\left(e\left(\frac{k}{n}+\frac{1}{2 n}\right)-e\left(\frac{j}{n}\right)\right)^{2}}=\sum_{j} \frac{e\left(\frac{k}{n}+\frac{j}{n}+\frac{1}{2 n}\right) E\left(\varepsilon_{j}\right)}{\left(e\left(\frac{k}{n}+\frac{1}{2 n}\right)-e\left(\frac{j}{n}\right)\right)^{2}}+O\left(n^{3} \varepsilon^{2}\right)$.

The sum on the left side equals $-\frac{n(n-2)}{4}$ because it equals

$$
-z^{2} \frac{d^{2}}{d z^{2}} \log \left(z^{n}-1\right)=\frac{n z^{2 n}+\left(n^{2}-n\right) z^{n}}{\left(z^{n}-1\right)^{2}}=\sum_{j} \frac{z^{2}}{\left(z-e\left(\frac{j}{n}\right)\right)^{2}}
$$

evaluated at $e\left(\frac{k}{n}+\frac{1}{2 n}\right)$. This brings us to

$$
\begin{aligned}
\frac{n}{2} E( & \left.-\alpha_{k}\right)-\frac{1}{4}\left(n^{2}-2 n\right) E\left(\alpha_{k}\right) \\
& =\sum_{j} \frac{E\left(\varepsilon_{j}\right)}{\left(e\left(\frac{k}{2 n}+\frac{1}{4 n}-\frac{j}{2 n}\right)-e\left(-\frac{k}{2 n}-\frac{1}{4 n}+\frac{j}{2 n}\right)\right)^{2}}+O\left(n^{3} \varepsilon^{2}\right) \\
& =-\sum_{j} \frac{E\left(\varepsilon_{j}\right)}{4 \sin ^{2}\left(2 \pi\left(\frac{k}{2 n}+\frac{1}{4 n}-\frac{j}{2 n}\right)\right)}+O\left(n^{3} \varepsilon^{2}\right) .
\end{aligned}
$$


Now use the approximation $E(t)=t+O\left(t^{2}\right)$, for $t$ small, and solve for $\alpha_{k}$ :

$$
\begin{aligned}
\alpha_{k} & =\frac{1}{n^{2}} \sum_{j} \frac{\varepsilon_{j}}{\sin ^{2}\left(2 \pi\left(\frac{k}{2 n}+\frac{1}{4 n}-\frac{j}{2 n}\right)\right)}+O\left(n \varepsilon^{2}\right) \\
& =\frac{1}{n^{2}} \sum_{j} \frac{\varepsilon_{j+k}}{\sin ^{2}\left(\frac{\pi}{2} \frac{2 j-1}{n}\right)}+O\left(n \varepsilon^{2}\right),
\end{aligned}
$$

as claimed.

4.3. Rate of convergence to equal spacing. Now we analyze the rate at which differentiation evens out zero spacings.

For both the midpoint and differentiation process, applying the operation twice has somewhat nicer properties than just applying it once. For example, if the points are equally spaced, then applying the operation twice leaves the sequence unchanged. In the discussion below we will generally work with the second derivative and second midpoint process, although the same ideas apply to the basic process.

We will view differentiation and midpoint averaging as two examples of a general procedure. Suppose $P$ is a probability measure on the integers such that $P$ is even, $P(0)$ is the maximum, and $P(j)$ is increasing for $j<0$ and decreasing for $j>0$. The conditions on $P$ are natural if one is thinking in terms of using $P$ to smooth out the irregularities of a sequence.

Given a sequence $\left(\varepsilon_{n}\right)$, define a sequence of sequences by $\beta_{n}^{0}=\varepsilon_{n}$ and

$$
\beta_{k}^{\ell+1}=\sum_{n} P(n) \beta_{n+k}^{\ell} .
$$

For the second derivative process $P(0)=\frac{1}{3}$ and $P(n)=\frac{2}{\pi^{2} n^{2}}$ otherwise, and for the (second) midpoint averaging process $P(0)=\frac{1}{2}, P(-1)=P(1)=\frac{1}{4}$, and $P(n)=0$ otherwise.

In Theorem 2.4.2 we start with $z_{k}=k+\varepsilon_{k}$, so in the notation above the discrepancy of $z_{n+1}^{\ell}$ and $z_{n}^{\ell}$ from the average spacing is $\beta_{k+1}^{\ell}-\beta_{k}^{\ell}$. We wish to estimate this in terms of $\varepsilon_{n}$. We can write $\beta_{k}^{\ell}$ in terms of $\varepsilon_{n}$ as, for instance,

$$
\beta_{k}^{\ell}=\sum_{n} P^{\ell}(n) \varepsilon_{n+k} .
$$

We can estimate the difference as

$$
\begin{aligned}
\beta_{k+1}^{\ell}-\beta_{k}^{\ell} & =\sum_{n} \varepsilon_{n+k}\left(P^{\ell}(n)-P^{\ell}(n-1)\right) \\
& \leq \varepsilon \sum_{n}\left|P^{\ell}(n)-P^{\ell}(n-1)\right| \\
& =2 \varepsilon P^{\ell}(0) .
\end{aligned}
$$

The last step requires that $P^{\ell}(0)$ is the maximum, and $P^{\ell}(n)$ is increasing for $n<0$ and decreasing for $n>0$. We will see that this is the case.

It can be seen that $P^{\ell}=P * P * \cdots * P$ is just the $\ell$-fold iterated convolution of $P$ with itself, where

$$
P * Q(n)=\sum_{m} P(m) Q(n-m) .
$$


The $\ell=2$ case was demonstrated in Section 4.1, where $\beta_{k}$ was calculated from $\alpha_{j}$. It is straightforward to check that if 0 is the global maximum for $P$ and $Q$, and both functions are increasing for $n<0$ and decreasing for $n>0$, then the same holds for $P * Q$. Thus, inequality (4.3.3) applies and we need only evaluate $P^{\ell}(0)=$ $P * P * \cdots * P(0)$.

The evaluation can be done using the properties of convolutions and Fourier transforms. Given a sequence $S$, let

$$
F_{S}(x)=\sum_{n} S(n) e^{2 \pi i n x}
$$

Then

$$
\begin{aligned}
F_{S}(x) F_{T}(x) & =\left(\sum_{n} S(n) e^{2 \pi i n x}\right)\left(\sum_{j} T(j) e^{2 \pi i j x}\right) \\
& =\sum_{n} \sum_{j} S(n) T(j) e^{2 \pi i(n+j) x} \\
& =\sum_{m} e^{2 \pi i m x} \sum_{n} S(n) T(m-n) \\
& =\sum_{m}(S * T)(m) e^{2 \pi i m x}
\end{aligned}
$$

That is, convolution of sequences corresponds to multiplication of Fourier series.

The final ingredient is to note that if

$$
F(x)=\sum_{j} c_{j} e^{2 \pi i j x}
$$

then

$$
c_{0}=\int_{0}^{1} F(x) d x
$$

provided we can integrate term by term, because $\int_{0}^{1} e^{2 \pi i j x} d x=0$ unless $j=0$. Thus,

$$
\begin{aligned}
P^{\ell}(0) & =P * P * \cdots * P(0) \\
& =\int_{0}^{1}\left(\sum_{n} P(n) e^{2 \pi i n x}\right)^{\ell} d x
\end{aligned}
$$

Now we need only identify the function in the integrand above.

In the case of the (second) midpoint process, we have

$$
\sum_{n} P(n) e^{2 \pi i n x}=\frac{1}{2}+\frac{1}{2} \cos (2 \pi x)
$$


You can either recognize the Beta integral or ask a computer algebra package to verify that

$$
\begin{aligned}
P^{\ell}(0) & =\int_{0}^{1}\left(\frac{1}{2}+\frac{1}{2} \cos (2 \pi x)\right)^{\ell} d x \\
& =\frac{2 \Gamma\left(\frac{3}{2}+\ell\right)}{(1+2 \ell) \sqrt{\pi} \Gamma(1+\ell)} \\
& \sim \frac{1}{\sqrt{\pi \ell}} \\
& =O\left(\ell^{-\frac{1}{2}}\right),
\end{aligned}
$$

which is the error term from Theorem 3.1 .1 assuming $\varepsilon_{n} \ll 1$.

In the case of the (second) differentiation process, it follows from the Fourier expansion

$$
4\left(x-\frac{1}{2}\right)^{2}=\frac{1}{3}+\frac{4}{\pi^{2}} \sum_{n=1}^{\infty} \frac{\cos (2 \pi n x)}{n^{2}}, \quad 0 \leq x \leq 1,
$$

that

$$
\sum_{n} P(n) e^{2 \pi i n x}=4\left(x-\frac{1}{2}\right)^{2}
$$

for $0 \leq x \leq 1$. Thus

$$
\begin{aligned}
P^{\ell}(0) & =\int_{0}^{1}\left(4\left(x-\frac{1}{2}\right)^{2}\right)^{\ell} d x \\
& =\frac{1}{1+2 \ell} \\
& =O\left(\ell^{-1}\right),
\end{aligned}
$$

as claimed in Theorem 2.4 .2

The discrepancy from equal spacing is much smaller for the differentiation process than for the midpoint process, and this is not surprising. Both processes average among neighboring points, and that averaging will be more effective if it takes place over a larger range. In the setup we have described, if $P$ has finite variance $\sigma^{2}$, then $F_{P}(x) \sim 1-2 \pi^{2} \sigma^{2} x^{2}$ for $x$ near 0 . Therefore

$$
\int_{-\delta}^{\delta} F_{P}(x)^{\ell} d x \sim \frac{1}{\sqrt{2 \pi \sigma^{2} \ell}}
$$

as we saw for the midpoint process. For the differentiation process we found that $F_{P}(x)=1-b|x|+O\left(x^{2}\right)$ for $x$ near 0 , where $b=4$. Therefore

$$
\int_{-\delta}^{\delta} F_{P}(x)^{\ell} d x \sim \frac{2}{b \ell}
$$

as in Theorem 2.4.2, Many other behaviors are possible. If $P$ has infinite variance but decreases like $n^{-A}$, then $F_{P}(x) \sim 1-a|x|^{\alpha}$ as $x \rightarrow 0$, where $\alpha=A-1$, and so

$$
\int_{-\delta}^{\delta} F_{P}(x)^{\ell} d x \sim \frac{2 \Gamma\left(\frac{1}{\alpha}\right)}{\alpha(a \ell)^{\frac{1}{\alpha}}} .
$$

Thus, we have averaging processes where the $\ell$ th iterate approaches equal spacing as fast as any given power of $\ell$. 
It should be noted that if one violates the conditions that $P(0)$ is a maximum and $P(n)$ increases (decreases) for $n<0(n>0)$, then the "averaging process" may not lead to equal spacing. For example, if $P(-1)=P(1)=\frac{1}{2}$, then the process leads to two interlaced sequences which separately approach equal spacing.

\section{Proof of Theorem 2.4.1}

We wish to show that if $f$ is an entire function that meets suitable additional conditions, then the $n$th derivative $f^{(n)}$, appropriately rescaled, approaches the cosine function. The real issue here is proving that the zeros approach equal spacing with sufficient uniformity. For if the zeros approach equal spacing, then the Hadamard product for $f^{(n)}$ can be seen to be close to $A e^{B x} \cos (C x+D)$ for some $A, B, C, D$. So we first show that the zeros of $f^{(n)}$ approach equal spacing, and then the proof is almost immediate.

Our method makes extensive use of the midpoint process we studied in this paper. It would be interesting to find a more direct proof, which could possibly lead to a stronger result. At the end of this section we also discuss the case that the zeros of $f$ lie near, but not necessarily on, a line.

5.1. Repeated differentiation leads to equal spaced zeros. We have shown that under repeated differentiation the small gaps between zeros are becoming larger, and the large gaps are becoming smaller, but it does not trivially follow that those gaps are approximately equal. Since we know that the midpoint process gives gaps which approach equal spacing, one possibility is to show that differentiation is better than midpoint at evening out the sequence:

Conjecture 5.1.1. Differentiation is better than midpoint. Suppose $f$ is an entire function of order 1 which is real on the real axis and has only real zeros, and suppose $z_{j}$ are the zeros of $f$, listed in increasing order. If $q<p$ are consecutive zeros of $f^{\prime}$, then

$$
\inf \frac{1}{2}\left(z_{n+2}-z_{n}\right) \leq p-q \leq \sup \frac{1}{2}\left(z_{n+2}-z_{n}\right) .
$$

A similar result should also hold when the spacing between zeros of $f$ varies slowly, in analogy to the relation between Theorem 2.3.1 and Theorem 2.3.2. Note that we interpret the right side of the above inequality as " $\infty$ " if $f$ has only finitely many zeros.

In contrast to Theorems 2.3.1 and 2.3.2 the conjecture is not true if instead we assume $p$ and $q$ are zeros of $f^{\prime}+a f$, for if $a$ is large, then $p$ and $q$ are very close to zeros of $f$. Also, the conjecture is not true if $\frac{1}{2}\left(z_{n+2}-z_{n}\right)$ is replaced by $\frac{1}{3}\left(z_{n+3}-z_{n}\right)$.

We now show that repeated differentiation leads to equal spaced zeros. The proof makes use of the fact that the midpoint process leads to equal spacing, but our approach is somewhat less elegant than would follow from the above conjecture.

Proposition 5.1.2. Suppose $f$ is an entire function which is real on the real line, has only real zeros, and $n_{+}(r) \sim n_{-}(r) \sim r$. Then if $\left(z_{n}^{j}\right)$ are the ordered zeros of the $j$ th derivative $f^{(j)}$, then $z_{n+1}^{j}-z_{n}^{j}=1+o(1)$ as $j \rightarrow \infty$. In addition, $\left|z_{n}^{j}\right| \gg n$.

Proof. The same method as in the proof of Theorems 2.3.1 and 2.3.2 shows that if $\left(z_{n}\right)$ are the ordered zeros of $f$ and $\left(z_{n}^{\prime}\right)$ are the ordered zeros of $f^{\prime}$, then

$$
\inf \left(z_{n+N}-z_{n}\right) \leq z_{n+N}^{\prime}-z_{n}^{\prime} \leq \sup \left(z_{n+N}-z_{n}\right),
$$


for any $N$, and similarly when considering zeros with $\left|z_{n}-z_{n}^{\prime}\right|<X$. When $N=2$, the difference $z_{n+2}-z_{n}$ is twice the gap between the midpoints of consecutive zeros of $f$. By Theorem 3.1.1, iterating the midpoint process gives sequences which approach equal spacing. Thus, the sequence of next nearest neighbors of zeros of $f^{(j)}$ approaches equal spacing. That is, the zeros of $f^{(j)}$ consist of two interlaced sequences which separately are approaching the (same) equal spacing.

It remains to show that the whole sequence of $f^{(j)}$ zeros is approaching equal spacing. One possibility is to now prove that $f^{(j)}(z)$ is approximately of the form $A e^{B z}(\cos (C z+D)+E)$, so then the zeros of derivatives of $f^{(j)}$ will approach equal spacing, as discussed in the paragraph after Theorem 2.4.1 But our goal is to prove that the zeros become equally spaced and then use that as a tool to prove the functional form.

We will show that the zeros of $f^{(j)}$ also consist of three interlaced sequences, each separately approaching equal spacing. From this it immediately follows that the entire sequence is approaching equal spacing. We will use (5.1.1) with $N=3$. Note that

$$
\begin{aligned}
z_{n+3}-z_{n} & =\left(z_{n+3}+z_{n+2}+z_{n+1}\right)-\left(z_{n+2}+z_{n+1}+z_{n}\right) \\
& =3\left(\tilde{z}_{n+2}-\tilde{z}_{n+1}\right)
\end{aligned}
$$

where $\tilde{z}_{n}=\frac{1}{3}\left(z_{n-1}+z_{n}+z_{n+1}\right)$. By either imitating the proof of Theorem 3.1 .1 or using the method of Section 4.3 with $P(-1)=P(0)=P(1)=\frac{1}{3}$, we see that iterating the averaging process $\left(z_{n}\right) \rightarrow\left(\tilde{z}_{n}\right)$ leads the sequence to approach equal spacing. So $z_{n+3}^{j}-z_{n}^{j}$ is approaching equal spacing, as required.

The final assertion follows from the fact that $z_{n}^{0} \sim n$ and that each successive zero set interlaces the previous one.

5.2. Proof of Theorem 2.4.1. Assume $\kappa=1$, so the zeros of $f^{(j)}$ are 1 apart on average. We first shift the function slightly so that the zeros are arranged conveniently. Suppose the smallest nonnegative zero of $f^{(j)}$ is at $w_{1}$ and the largest negative zero is at $w_{-1}$. Choose $d_{j}=\frac{1}{2}\left(w_{-1}+w_{1}\right)$ and let $z_{1}, z_{2}, \ldots$ denote the positive zeros of $f^{(j)}\left(z+d_{j}\right)$ and $z_{-1}, z_{-2}, \ldots$ the negative zeros.

We have the Hadamard factorization

$$
\begin{aligned}
f^{(j)}\left(z+d_{j}\right)= & A_{j} \exp \left(B_{j} z\right) \prod_{n}\left(1-\frac{z}{z_{n}}\right) \exp \left(\frac{z}{z_{n}}\right) \\
= & A_{j} \exp \left(B_{j}^{\prime} z\right) \prod_{1 \leq n \leq Y}\left(1-\frac{z}{z_{-n}}\right)\left(1-\frac{z}{z_{n}}\right) \\
& \times \prod_{|n|>Y}\left(1-\frac{z}{z_{n}}\right) \exp \left(\frac{z}{z_{n}}\right) \\
= & A_{j} \exp \left(B_{j}^{\prime} z\right) F_{j}(z) G_{j}(z),
\end{aligned}
$$

say, where $Y$ is to be chosen later. We must show that if $|z|<X$, then $F_{j}(z) \rightarrow$ $\cos (\pi z)$ and $G_{j}(z) \rightarrow 1$.

Given $X, \varepsilon>0$, first choose $Y$ so that $\left|G_{j}(z)-1\right|<\varepsilon e^{-\pi X}$ for $|z|<X$ and all $j>0$. This is possible because $z_{n} \gg n$.

Let $F$ and $G$ denote the above products in the special case that $z_{ \pm n}=\left(n \mp \frac{1}{2}\right)^{-1}$ for all $n>0$. Since $F(z) G(z)=\cos (\pi z)$, it follows that $|\cos (\pi z)-F(z)|<\varepsilon$ for $|z|<X$. 
For each $n$, as $j \rightarrow \infty$ the gap $z_{n}^{j}-z_{n-1}^{j}$ is approaching 1 , so we can a choose $j$ so that $z_{ \pm n}$ is sufficiently close to $\left(n \mp \frac{1}{2}\right)^{-1}$ for $0<n \leq Y$ to ensure that $\left|F_{j}(z)-F(z)\right|<\varepsilon$ for $|z|<X$. Using $|\cos (\pi z)|<e^{\pi X}$ for $|z|<X$ and combining all estimates we have $\left|A_{j}^{-1} \exp \left(-B_{j}^{\prime} z\right) f^{(j)}\left(z+D_{j}\right)-\cos (\pi z)\right|<4 \varepsilon$ for $|z|<X$, which completes the proof.

5.3. Zeros not on a line. We have seen that repeated differentiation leads to a function whose zeros approach equal spacing. Surprisingly, this does not always require the zeros of the original function to lie on a line (or circle), but only that the zeros lie in a suitable neighborhood of the line (or circle).

This is easy to see in the case of the circle. Suppose $f$ is a degree $n$ polynomial and let $g(z)=z^{-n / 2} f(z)$, so

$$
g(z)=a_{n} z^{\frac{n}{2}}+a_{n-1} z^{\frac{n}{2}-1}+\cdots+a_{0} z^{-\frac{n}{2}} .
$$

Then

$$
z g^{\prime}(z)=\frac{n}{2} a_{n} z^{\frac{n}{2}}+\left(\frac{n}{2}-1\right) a_{n-1} z^{\frac{n}{2}-1}+\cdots+\left(-\frac{n}{2}\right) a_{0} z^{-\frac{n}{2}},
$$

and the " $k$ th derivative" equals

$$
\left(z \frac{d}{d z}\right)^{k} g(z)=\left(\frac{n}{2}\right)^{k} a_{n} z^{\frac{n}{2}}+\left(\frac{n}{2}-1\right)^{k} a_{n-1} z^{\frac{n}{2}-1}+\cdots+\left(-\frac{n}{2}\right)^{k} a_{0} z^{-\frac{n}{2}}
$$

If $a_{0} \neq 0$, then the factors $\left(\frac{n}{2}\right)^{k}$ from the first and last terms dominate everything else, so the zeros are approaching the zeros of $z^{n}+(-1)^{k} \frac{a_{0}}{a_{n}}$. Note that we did not actually require the zeros of the original polynomial $f$ to be on the unit circle. As long as 0 is not a root of $f$, the above process gives a sequence of functions whose zeros eventually lie on a circle, and in fact approach equal spacing on a circle!

For zeros on a line, we have the following result of Young-One Kim (this is a slight revision of Theorem 2 from [12]):

Theorem 5.3.1. Let $f(z)$ be a nonconstant real entire function, $0<\rho \leq 2$, and assume that $f(z)$ is of order less than $\rho$ or is of order $\rho$ and minimal type. If there is a positive real number $A$ such that all the zeros of $f(z)$ are distributed in the infinite strip $|\Im z| \leq A$, then for any positive constant $B$ there is a positive integer $n_{1}$ such that $f^{(n)}(z)$ has only real zeros in $|\Re z| \leq B n^{\frac{1}{\rho}}$ for all $n \geq n_{1}$.

In other words, if the zeros do not start out too far from the real axis, then they end up on the real axis as you differentiate. Thus, Theorem 2.4.1 can be modified to only assume that the zeros lie in a strip around the real axis, and Conjecture 2.4.4 should only require the zeros to lie near the real axis.

The theorem applies to the Riemann $\Xi$-function with $\rho=1+\varepsilon$. It is interesting to note that Conrey [2] has shown that $\Xi^{(j)}$ has a positive proportion of its zeros on the real axis, and that proportion is $1+O\left(j^{-2}\right)$ as $j \rightarrow \infty$.

If $f(z)=\sum_{j=0}^{\infty} c_{j} x^{j} / j$ ! is entire of order 1 and all $c_{j}$ are real, then a necessary condition for the zeros of $f$ to be real is the Turán inequalities $c_{k}^{2}-c_{k-1} c_{k+1} \geq 0$, for $k \geq 1$. The above ideas suggest that the Turán inequalities should hold for sufficiently large $k$, provided only that the zeros of $f$ lie in a neighborhood of the real axis. 


\section{REFERENCES}

[1] R.P. Boas, Entire Functions, Academic Press, 1954. MR 0068627 (16:914f)

[2] J.B. Conrey, Zeros of derivatives of Riemann's $\xi$-function on the critical line, J. Number Theory 16 (1983), no. 1, 49-74. MR0693393 (84g:10070)

[3] J.B. Conrey, D.W. Farmer, J. Keating, M. Rubinstein, and N.C. Snaith, Integral moments of families of L-functions, http://arxiv.org/abs/math.NT/0206018.

[4] J.B. Conrey and H. Iwaniec, Spacing of zeros of Hecke L-functions and the class number problem, Acta Arith. 103 (2002) no. 3, 259-312. MR1905090 (2003h:11103)

[5] T. Craven, G. Csordas, and W. Smith, Zeros of derivatives of entire functions, PAMS 101 (1987), 323-326. MR0902550 (88k:30024)

[6] T. Craven, G. Csordas, and W. Smith, Zeros of derivatives of entire functions and the PólyaWiman conjecture, Ann. of Math (2) 125 (1987), 405-431. MR0881274 (88a:30007)

[7] P. Davis, Circulant Matrices, John Wiley and Sons, 1979. MF0543191 (81a:15003)

[8] D.W. Farmer and S.M. Gonek, Pair correlation of the zeros of $\xi^{\prime}$, in preparation.

[9] H. Ki, personal communication.

[10] H. Ki and Y.-O. Kim, On the number of nonreal zeros of real entire functions and the FourierPólya conjecture, Duke Math. J, Vol. 104, No. 1, (2000), 45-73. MR.1769725 (2001h:30022)

[11] Y.-O. Kim, A proof of the Pólya-Wiman conjecture, PAMS 109 (1990), 1045-1052. MR.1013971 (90k:30049)

[12] Y.-O. Kim, Critical points of real entire functions and a conjecture of Pólya, PAMS, Vol. 124, No. 3, (1996), 819-829. MR.1301508 (96f:30027)

[13] R. Kreminski, Newton-Cotes integration for approximating Stieltjes (generalized Euler) constants. Math. Comp. 72 (2003), no. 243, 1379-1397. MR.1972742 (2004a:11140)

[14] R. Kreminski, http://www.tamu-commerce.edu/coas/math/FACULTY/KREMIN/ stieltjesrelated/

[15] N. Levinson and H. Montgomery, Zeros of the derivatives of the Riemann zeta-function, Acta Math. 133 (1974), 49-65. MR 0417074 (54:5135)

[16] G. Pólya, Some problems connected with Fourier's work on transcendental equations, Quart. J. Math. Oxford Ser. (2) 1 (1903), 21-34.

[17] T. Sheil-Small, On the zeros of the derivatives of real entire functions and Wiman's conjecture. Ann. of Math. (2) 129 (1989), no. 1, 179-193. MR0979605 (90a:30084)

[18] A. Stoyanoff, Sur un Thórem de M. Marcel Riesz, Nouvelles Annales de Mathématique, 1 (1926), 97-99.

[19] J.v. Sz.-Nagy, Über Polynome mit lauter reelen Nullstellen, Acta Math. Acad. Scient. Hung. 1 (1950), 225-8. MR0046479(13:740f)

[20] P. Walker, Separation of the zeros of polynomials, Amer. Math. Monthly 100 (1993), no. 3, 272-273. MR 1212834

[21] P. Walker, Bounds for the separation of real zeros of polynomials, J. Austral. Math. Soc. (Series A) 59 (1995), 330-342. MR.1355224 (96i:26017)

American Institute of Mathematics, 360 Portage Avenue, Palo Alto, California 94306-2244

E-mail address: farmer@aimath.org

Department of Mathematics, Bucknell University, Lewisburg, Pennsylvania 17837

E-mail address: rrhoades@bucknell.edu 